\title{
From the Kinematics of Precession Motion to Generalized Rabi Cycles
}

\author{
Danail S. Brezov, ${ }^{1}$ Clementina D. Mladenova, ${ }^{2}$ and Ivaïlo $M$. Mladenov $\left(\mathbb{D}{ }^{3}\right.$ \\ ${ }^{1}$ University of Architecture, Civil Engineering and Geodesy, 1 Hristo Smirnenski Blvd., 1046 Sofia, Bulgaria \\ ${ }^{2}$ Institute of Mechanics, Bulgarian Academy of Sciences, Acad. G. Bonchev Str., Block 4, 1113 Sofia, Bulgaria \\ ${ }^{3}$ Institute of Biophysics and Biomedical Engineering, Bulgarian Academy of Sciences, Acad. G. Bonchev Str., \\ Block 21, 1113 Sofia, Bulgaria
}

Correspondence should be addressed to Ivaillo M. Mladenov; mladenov@bio21.bas.bg

Received 29 September 2017; Accepted 16 January 2018; Published 28 February 2018

Academic Editor: Manuel Calixto

Copyright (C) 2018 Danail S. Brezov et al. This is an open access article distributed under the Creative Commons Attribution License, which permits unrestricted use, distribution, and reproduction in any medium, provided the original work is properly cited.

\begin{abstract}
We use both vector-parameter and quaternion techniques to provide a thorough description of several classes of rotations, starting with coaxial angular velocity $\boldsymbol{\Omega}$ of varying magnitude. Then, we fix the magnitude and let $\boldsymbol{\Omega}$ precess at constant rate about the $z$-axis, which yields a particular solution to the free Euler dynamical equations in the case of axially symmetric inertial ellipsoid. The latter appears also in the description of spin precessions in NMR and quantum computing. As we show below, this problem has analytic solutions for a much larger class of motions determined by a simple condition relating the polar angle and $z$-projection of $\boldsymbol{\Omega}$ (expressed in cylindrical coordinates), which are both time-dependent in the generic case. Relevant physical examples are also provided.
\end{abstract}

\section{Introduction: Quaternions and Vector-Parameters}

The Clifford algebra of quaternions $\mathbb{H} \cong C \ell_{0,2}(\mathbb{R}) \cong C \ell_{3}^{0}(\mathbb{R})$ is known to be intimately related to the rotation group in $\mathbb{R}^{3}$ and one way to see it is via the standard spin covering map $\operatorname{Spin}(3) \cong \mathrm{SU}(2) \stackrel{\mathbb{Z}_{2}}{\longrightarrow} \mathrm{SO}(3)$ that is topologically a projection from the unit sphere $\mathbb{S}^{3} \stackrel{\mathbb{Z}_{2}}{\longrightarrow} \mathbb{R P}^{3}$. The basis of bivectors in $\mathrm{Cl}_{3}$ is spanned by three units satisfying

$$
\mathbf{i}^{2}=\mathbf{j}^{2}=\mathbf{k}^{2}=\mathbf{i j k}=-\mathbf{1},
$$

where 1 denotes the identity element, so one can express each

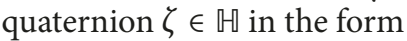

$$
\zeta=\left(\zeta_{0}, \zeta\right)=\zeta_{0} \mathbf{1}+\zeta_{1} \mathbf{i}+\zeta_{2} \mathbf{j}+\zeta_{3} \mathbf{k}
$$

Hence, as a linear space, $\mathbb{H}$ may obviously be identified with $\mathbb{R}^{4} \cong \mathbb{R} \times \mathbb{R}^{3}$ by introducing coordinates as shown above. At the same time, quaternions have a specific Clifford multiplication rule that can be expressed in the above notation as

$$
\left(\zeta_{0}, \zeta\right)\left(\widetilde{\zeta}_{0}, \widetilde{\zeta}\right)=\left(\zeta_{0} \widetilde{\zeta}_{0}-\zeta \cdot \widetilde{\zeta}, \zeta_{0} \widetilde{\zeta}+\widetilde{\zeta}_{0} \zeta+\zeta \times \widetilde{\zeta}\right)
$$

where and $\times$ stand for the dot and cross products in $\mathbb{R}^{3}$, respectively. Just like in the case of complex numbers, Clifford conjugation in $\mathbb{H}$ is given by sign inversion of the imaginary (vector) part $\zeta$ : that is, $\zeta=\left(\zeta_{0}, \zeta\right) \rightarrow \zeta^{*}=\left(\zeta_{0},-\zeta\right)$, and since there are no zero divisors, the quaternion norm defined as $\|\zeta\|^{2} \mathbf{1}=\zeta^{*} \zeta$ yields the inverse of every nonzero element $\zeta^{-1}=\|\zeta\|^{-2} \zeta^{*}$. In particular, restricting to $\|\zeta\|^{2}=1$, one obtains the unit sphere in $\mathbb{R}^{4}$, which the composition law (3) endows with the group structure of $\mathrm{SU}(2)$. At this point, rotations in $\mathbb{R}^{3}$ appear quite naturally if we identify threevectors with pure (imaginary) quaternions $\mathbf{x} \in \mathbb{R}^{3} \rightarrow \widetilde{X}=$ $x_{1} \mathbf{i}+x_{2} \mathbf{j}+x_{3} \mathbf{k} \in \mathfrak{g} \mathfrak{u}(2)$ and use the adjoint action of the spin group $\operatorname{Ad}_{\zeta}: \widetilde{X} \rightarrow \zeta \widetilde{X} \zeta^{*}$. This effectively transforms the components of $\mathbf{X}$ via the linear orthogonal map

$$
\mathscr{R}(\zeta)=\left(\zeta_{0}^{2}-\zeta^{2}\right) \mathscr{I}+2 \zeta \zeta^{t}+2 \zeta_{0} \zeta^{\times},
$$


where $\mathscr{I}$ denotes the identity in $\mathbb{R}^{3}, \zeta \zeta^{t}$ stands for the usual dyadic (tensor) product, and $\zeta^{\times}$is the skew-symmetric linear map associated with $\zeta$ via the Hodge duality: that is, $\zeta^{\times} \widetilde{\zeta}=\zeta \times \widetilde{\zeta}$. The above map clearly preserves orientation and thus represents rotation in $\mathbb{R}^{3}$. Denoting its angle $\phi$ and the unit vector along its axis $\mathbf{n}$, one may express the associated quaternion as

$$
\begin{aligned}
\zeta_{0} & =\cos \frac{\phi}{2} \\
\zeta & =\sin \frac{\phi}{2} \mathbf{n} .
\end{aligned}
$$

Central projection onto the plane $\zeta_{0}=1$ then yields the vector-parameter $\mathbf{c}=\zeta / \zeta_{0}=\tan (\phi / 2) \mathbf{n}$ that is actually not a vector but rather a point in projective three-space, equipped with an additional group structure. This simple construction appears quite convenient: on the one hand, unlike other known alternatives (such as Euler angles which can be considered as coordinates of points in projective space on the three-torus and lead to singularities, e.g., the so-called gimbal lock) it gives a topologically adequate description of the orthogonal group $\mathrm{SO}(3) \cong \mathbb{R} \mathbb{P}^{3}$, while, on the other hand, it provides an efficient associative composition law

$$
\left\langle\mathrm{c}_{2}, \mathrm{c}_{1}\right\rangle=\frac{\mathrm{c}_{2}+\mathrm{c}_{1}+\mathrm{c}_{2} \times \mathrm{c}_{1}}{1-\mathrm{c}_{2} \cdot \mathrm{c}_{1}}
$$

inherited from the quaternion multiplication (3) upon central projection. This constitutes a (nonlinear) representation of $\mathrm{SO}(3)$ related to the usual matrix realization via $\mathscr{R}\left(\left\langle\mathbf{c}_{2}, \mathbf{c}_{1}\right\rangle\right)=$ $\mathscr{R}\left(\mathbf{c}_{2}\right) \mathscr{R}\left(\mathbf{c}_{1}\right)$, in which the inverse element is given by $-\mathbf{c}$ and the neutral one by the zero vector. Moreover, it yields rational expressions for the matrix entries of $\mathscr{R}(\mathbf{c})$ that may be obtained directly from (4) or equivalently by means of the famous Cayley transform

$$
\mathscr{R}(\mathbf{c})=\frac{\mathscr{I}+\mathbf{c}^{\times}}{\mathscr{I}-\mathbf{c}^{\times}}=\frac{\left(1-\mathbf{c}^{2}\right) \mathscr{I}+2 \mathbf{c c}^{t}+2 \mathbf{c}^{\times}}{1+\mathbf{c}^{2}} .
$$

Clearly, the above follows also from Rodrigues' rotation formula with the aid of Euler's trigonometric substitution, but the projective approach reveals the major advantages of vector parameterization in a much more direct manner. Recall also that the angular velocity of a rotating rigid body is defined in the body and inertial frames, respectively, as

$$
\begin{aligned}
\boldsymbol{\Omega}^{\times} & =\dot{\mathscr{R}} \mathscr{R}^{t}, \\
\boldsymbol{\omega}^{\times} & =\mathscr{R}^{t} \dot{\mathscr{R}} .
\end{aligned}
$$

Straightforward differentiation of the Cayley representation (7) yields the relations (see also $[1,2]$ )

$$
\begin{aligned}
& \boldsymbol{\Omega}=\frac{2}{1+\mathbf{c}^{2}}\left(\mathscr{I}+\mathrm{c}^{\times}\right) \dot{\mathbf{c}}, \\
& \boldsymbol{\omega}=\frac{2}{1+\mathbf{c}^{2}}\left(\mathscr{I}-\mathbf{c}^{\times}\right) \dot{\mathbf{c}}
\end{aligned}
$$

while in the inverse direction, one easily derives the Riccati equations for the vector-parameter

$$
\dot{\mathbf{c}}=\frac{1}{2}\left(\mathscr{I}+\mathrm{cc}^{t}-\mathrm{c}^{\times}\right) \boldsymbol{\Omega}=\frac{1}{2}\left(\mathscr{I}+\mathrm{cc}^{t}+\mathrm{c}^{\times}\right) \boldsymbol{\omega} .
$$

We refer to [3] for a detailed introduction to quaternions and some of their applications in a wide range or fields, from navigation and attitude control to computer graphics. In quantum mechanics quaternions and Pauli matrices appear naturally in the description of spin systems as we recall below. Moreover, modern physics embraces the idea of quaternionic Hilbert space and quaternionic quantum mechanics (cf. [4, 5]). Many features of complex numbers are preserved except, of course, commutativity, which makes the definition of quaternionic analyticity far nontrivial and yet fruitful (see [6]), for example, in the description of electrodynamics [7] and its relation to the so-called Fueter analyticity. Vector parameterization $[8,9]$, on the other hand, is much less popular although it has proven quite efficient in the description of (pseudo-)orthogonal groups in dimensions three and four [10], as well as in a wide range of problems related to classical and quantum mechanics $[2,11]$, special relativity and electrodynamics $[12,13]$, robotics and navigation $[3,14]$, and various other fields of science and technology. Here we make use of both descriptions.

The text is organized as follows: after this brief introductory part, we consider the kinematical problem of a rotating rigid body with fixed direction of the angular velocity vector using the projective vector-parameter construction discussed above, thus extending an old result due to O'Reilly and Payen [15]. Then, in Section 3, we switch back to quaternions, which lead to linear, rather than Riccati equations, in order to deal with the more complicated settings. Namely, we consider the case of a precessing (about a fixed axis) angular velocity and the most general case, given conveniently in cylindrical coordinates, using techniques from the arsenal of quantum mechanics, which allow us to derive a simple integrability condition. Finally, after a brief note on dynamics, we relate these results to the Rabi oscillator that appears in quantum computation, showing how our solutions yield more freedom in the entire process.

\section{The Coaxial Angular Velocity (CAV) Motion}

Now, let us consider the first equation in (10) and look for solutions with a fixed direction of $\boldsymbol{\Omega}$. Without loss of generality, we may choose for convenience a reference frame, in which $\boldsymbol{\Omega}$ is aligned with the $z$-axis: that is,

$$
\boldsymbol{\Omega}=(0,0, \Omega)^{t}, \quad \Omega=2 \omega(t) .
$$

This allows for expressing the matrix ODE (10) explicitly in components as

$$
\begin{aligned}
& \dot{c}_{1}=\omega\left(c_{1} c_{3}-c_{2}\right), \\
& \dot{c}_{2}=\omega\left(c_{2} c_{3}+c_{1}\right), \\
& \dot{c}_{3}=\omega\left(1+c_{3}^{2}\right) .
\end{aligned}
$$


From the third equation, one obviously has (we set the initial moment at $t=0$ )

$$
c_{3}(t)=\tan \vartheta, \quad \vartheta(t)=\dot{\vartheta}+\int_{0}^{t} \omega(s) \mathrm{d} s,
$$

while for the first two we may use the substitution $z(t)=$ $c_{1}(t)+\mathrm{i} c_{2}(t)$, which yields

$$
\dot{z}(t)=\left(c_{3}(t)+\mathrm{i}\right) \omega(t) z(t) .
$$

The latter clearly possesses a trivial solution $z \equiv 0$ that is a well-known result: in this case, the rotation takes place about the axis determined by the angular velocity vector $\Omega$ with magnitude $\Omega=2 \omega$. However, for $z \neq 0$, one has

$$
\frac{\mathrm{d}}{\mathrm{d} t}(\ln z)=(\tan \vartheta+\mathrm{i}) \omega
$$

Combining the above two cases, one may express the general solution in the form (here the amplitude $\rho \geq 0$ and the two phases $\stackrel{\circ}{\varphi}, \stackrel{0}{\vartheta} \in[0,2 \pi)$ are constants of integration)

$$
z(t)=\rho \sec \vartheta \mathrm{e}^{\mathrm{i} \varphi}, \quad \varphi(t)=\dot{\varphi}+\int_{0}^{t} \omega(s) \mathrm{d} s
$$

from which it is straightforward to write

$$
\mathbf{c}(t)=\sec \vartheta(\rho \cos \varphi, \rho \sin \varphi, \sin \vartheta)^{t} .
$$

Changing the parameter $t \rightarrow \tau=\tan \vartheta(t)$, it is not hard to show that the integral curves (17) obtained above are actually rays in $\mathbb{R}^{3}$ described by

$$
\mathbf{c}(\tau)=(u+v \tau, u \tau-v, \tau)^{t}, \quad \tau \in \mathbb{R} \mathbb{P}^{1}
$$

where the constants $u$ and $v$ are most naturally given in polar coordinates as

$$
\begin{aligned}
& u=\rho \cos (\dot{\vartheta}-\dot{\varphi}), \\
& v=\rho \sin (\dot{\vartheta}-\dot{\varphi}) .
\end{aligned}
$$

In this way, we end up with a two-parameter family of rays in $\mathbb{R}^{3}$, whose intersection points $(x, y, \tilde{x}, \tilde{y})=(u,-v, u+v, u-v)$ with the planes $z=0$ and $z=1$ constitute isosceles right triangles with the origin. In other words, if we denote $\mathbf{c}(\tau)=$ $\mathbf{c}_{0}+\tau \mathbf{v}$ in (18), the constant vectors $\mathbf{c}_{0}$ and $\mathbf{v}$ are perpendicular and $\mathbf{c}_{0}^{2}=\mathbf{v}_{0}^{2}$, where $\mathbf{v}_{0}=\mathbf{v}-\widehat{\mathbf{e}}_{z}$ is the projection of $\mathbf{v}$ in the $(x, y)$-plane. Those are all orbits still periodic in $\vartheta$. In particular, the vertical $z$-axis and the horizontal projective line at infinity are limiting cases corresponding to $\rho \rightarrow 0$ and $\rho \rightarrow \infty$ as discussed below in detail. Note that one may redefine $\dot{\varphi}$ via an overall phase shift introduced by a proper choice of initial time, which yields $\stackrel{\circ}{\mathcal{Y}}=0$, so in physical terms one has only two relevant constants (amplitude and phase) and the general solution of (12) is generated by the time flow of $\mathbf{c}(t)$ as orbits of all possible initial points. On the other hand, it is not difficult to see that the whole manifold $\mathrm{SO}(3) \cong \mathbb{R} \mathbb{P}^{3}$ is covered by the above map. Moreover, each point in projective space is reached by the initial data setting $t=0$ since $\mathbb{R} \mathbb{P}^{3}$ is obviously covered by the family of rays (18), in which we may use $\stackrel{\ominus}{\vartheta}$ as an intrinsic parameter. Now, in order to lift the whole construction up to the spin cover, we may express the two images $\zeta^{ \pm}=\pi_{ \pm}^{-1} \mathbf{c} \in \mathbb{S}^{3}$ corresponding to the vector-parameter (17) in the form

$$
\zeta^{ \pm}= \pm\left(\begin{array}{cc}
\sin \psi \mathrm{e}^{\mathrm{i} \varphi} & \mathrm{i} \cos \psi \mathrm{e}^{-\mathrm{i} \vartheta} \\
\mathrm{i} \cos \psi \mathrm{e}^{\mathrm{i} \vartheta} & \sin \psi \mathrm{e}^{-\mathrm{i} \varphi}
\end{array}\right)
$$

with a suitable choice of a quaternion basis and using the notation

$$
\psi=\arctan \rho \in\left[0, \frac{\pi}{2}\right]
$$

Note that the 9 -orbits on the unit sphere project to circles in the $(x, y)$ and $(z, w)$ planes with equal frequencies but generally different phases and amplitudes. In particular, for $\psi=0$ and $\psi=\pi / 2$ the former (resp., the latter) circle shrinks to a point. From the perspective of the Hopf map, one has quaternion $\vartheta$-orbits on $\mathbb{S}^{3}$ whose holonomy shifts the $\mathbb{S}^{1}$ phase $\vartheta$ by a whole period for each revolution about the $z$-axis of the base $\mathbb{S}^{2}$. This becomes more apparent if we write $\zeta$ with its coordinates on $\mathbb{S}^{3}$ as

$$
\left(\zeta^{ \pm}, \zeta_{0}^{ \pm}\right)= \pm(\sin \psi \cos \varphi, \sin \psi \sin \varphi, \cos \psi \sin \vartheta, \cos \psi \cos \vartheta)
$$

Stereographic projection (from the south pole onto the equatorial plane) yields also the so-called Wiener-Milenkovic conformal rotation vector (see [9] for more details)

$$
\boldsymbol{\varsigma}=\frac{\zeta}{1+\zeta_{0}}=\tan \frac{\phi}{4} \mathbf{n}
$$

which does not involve infinities at least for $\psi \neq 0$, so one may plot the corresponding curves in Euclidean space. Moreover, the rotation angle is not difficult to derive from formula (17): namely,

$$
\phi(t)=2 \arctan \left(\sec \vartheta \sqrt{\rho^{2}+\sin ^{2} \vartheta}\right) .
$$

As for the corresponding invariant axis, ignoring the common prefactor in (17), we see that it precesses about the vector $\boldsymbol{\Omega}$. Besides, the rate of precession and nutation are synchronized and in the case $\omega=$ const., equal to half the magnitude of the angular velocity $\Omega=2 \omega$. It is convenient also to introduce spherical coordinates for the unit invariant vector $\mathbf{n}=\mathbf{n}(\theta, \varphi)$ in the form

$$
\theta=\arctan (\rho \csc \vartheta)
$$

Using formula (7) and taking into account (21), we express the corresponding rotation matrix explicitly as 


$$
\mathscr{R}(\varphi, \vartheta, \psi)=\left(\begin{array}{ccc}
\cos 2 \vartheta \cos ^{2} \psi+\cos 2 \varphi \sin ^{2} \psi & \sin 2 \varphi \sin ^{2} \psi-\sin 2 \vartheta \cos ^{2} \psi & \sin (\vartheta+\varphi) \sin 2 \psi \\
\sin 2 \varphi \sin ^{2} \psi+\sin 2 \vartheta \cos ^{2} \psi & \cos 2 \vartheta \cos ^{2} \psi-\cos 2 \varphi \sin ^{2} \psi & -\cos (\vartheta+\varphi) \sin 2 \psi \\
\sin (\vartheta-\varphi) \sin 2 \psi & \cos (\vartheta-\varphi) \sin 2 \psi & \cos 2 \psi
\end{array}\right)
$$

Note that the angles $\varphi$ and $\vartheta$ differ only by a phase

$$
\vartheta=\varphi+\stackrel{\circ}{\vartheta}-\stackrel{\circ}{\varphi}
$$

while $\psi$ is a constant of integration, which makes the time dependence of the above matrix entries relatively simple. Moreover, we can manipulate both $\psi$ and $\vartheta-\varphi$ via specific choice of initial data to obtain some particular cases. Firstly, we discuss the three distinct types of solutions pointed out in [15] for the case $\omega=$ const. The most obvious one, for which c $\| \boldsymbol{\Omega}$ with $\Omega=\dot{\phi}$ (Type I), corresponds to $\psi \equiv 0(\rho \rightarrow 0)$ and is usually referred to as steady rotation. It suggests a constant invariant axis oriented along the angular velocity vector and linearly evolving angle $\phi$ : namely,

$$
\mathscr{R}_{\|}(\vartheta)=\left(\begin{array}{ccc}
\cos 2 \vartheta & -\sin 2 \vartheta & 0 \\
\sin 2 \vartheta & \cos 2 \vartheta & 0 \\
0 & 0 & 1
\end{array}\right) \text {. }
$$

On the other hand, setting $\psi=\pi / 2$, that is, $\rho \rightarrow \infty$, we obtain the half-turn example considered in [15] (Type II rotations) as $\mathscr{R}_{\perp}(\varphi)=2 \mathbf{n n}^{t}-\mathscr{I}$, where $\mathbf{n}=(\cos \varphi, \sin \varphi, 0)^{t}$, which may be written explicitly in matrix terms directly from (26) in the form (note that the 9 -dependence in (26) is completely factored out by demanding $\psi$ to be a right angle)

$$
\mathscr{R}_{\perp}(\varphi)=\left(\begin{array}{ccc}
\cos 2 \varphi & \sin 2 \varphi & 0 \\
\sin 2 \varphi & -\cos 2 \varphi & 0 \\
0 & 0 & -1
\end{array}\right) \text {. }
$$

This time the rotation takes place about a varying axis that remains perpendicular to $\Omega$ and precesses about it, while the angle remains $\phi=\pi$ at each particular instant of time.

The third type of rotations proposed in [15], in which both the rotation axes and angle are periodic functions of time, is also given by formula (26) if we simply set $\omega=$ const. We may consider, for example, the case of coherent phases $\vartheta=\varphi$, that is, $\stackrel{\circ}{\vartheta}=\stackrel{\circ}{\varphi}$, in which

$$
\mathbf{c}=(\rho, \rho \tan \varphi, \tan \varphi)^{t}
$$

and express the respective rotational matrix explicitly: that is,

$$
\begin{aligned}
& \mathscr{R}_{0}(\varphi, \psi) \\
& =\left(\begin{array}{ccc}
\cos 2 \varphi & -\sin 2 \varphi \cos 2 \psi & \sin 2 \varphi \sin 2 \psi \\
\sin 2 \varphi & \cos 2 \varphi \cos 2 \psi & -\cos 2 \varphi \sin 2 \psi \\
0 & \sin 2 \varphi & \cos 2 \psi
\end{array}\right) .
\end{aligned}
$$

Specific solutions for different values of the phase shift are constructed similarly. Note also that the above matrices have such a simple form only in the reference frame, in which the angular velocity $\Omega$ is aligned with one of the axes. Otherwise, the vector-parameter (17) needs to be rotated correspondingly, relative to the coordinate change.

The compact explicit form of the solution (26) allows for expressing the three parameters $\varphi, \vartheta$, and $\psi$, which we use as a substitute for the usual Euler angles, in the generic case $\psi \neq 0, \pi / 2$ as

$$
\begin{aligned}
\varphi & =-\frac{1}{2}\left[\operatorname{atan}_{2}\left(r_{31}, r_{32}\right)+\operatorname{atan}_{2}\left(r_{13}, r_{23}\right)\right], \\
\vartheta & =\frac{1}{2}\left[\operatorname{atan}_{2}\left(r_{31}, r_{32}\right)-\operatorname{atan}_{2}\left(r_{13}, r_{23}\right)\right], \\
\psi & =\frac{1}{2} \arccos r_{33},
\end{aligned}
$$

where $\operatorname{atan}_{2}$ stands for the proper quadrant inverse tangent (note that $\psi \in[0, \pi / 2]$, so $\sin 2 \psi \geq 0$ and $\cos 2 \psi$ is monotonous, thus invertible in this interval) and the $r_{j k}$ 's denote the matrix entries of $\mathscr{R}(\varphi, \vartheta, \psi)$ given by formula (26) in the standard basis. Moreover, since

$$
1+c^{2}=\left(1+\rho^{2}\right)\left(1+\tan ^{2} \vartheta\right)
$$

as it follows from formula (17), it is straightforward to obtain also the azimuthal angle $\theta$ in the spherical representation of the invariant unit vector $\mathbf{n}$ and the angle of rotation $\phi$, respectively, as

$$
\begin{aligned}
& \theta=\arctan (\csc \vartheta \tan \psi), \\
& \phi=2 \arccos (\cos \vartheta \cos \psi) .
\end{aligned}
$$

However, in the limiting cases of vanishing and infinite amplitude of precession, corresponding to $\psi=0$ and $\psi=$ $\pi / 2$, respectively, (32) yield indeterminacy, so these two cases need to be treated separately. In particular, for $\rho \rightarrow 0$, one has

$$
\begin{aligned}
& \psi=0 \Longrightarrow \vartheta=\frac{1}{2} \operatorname{atan}_{2}\left(r_{21}, r_{11}\right) \\
& \theta=0 \\
& \phi=2 \vartheta
\end{aligned}
$$

while the polar angle $\varphi$ becomes obsolete. Similarly, the limit $\rho \rightarrow \infty$ yields 


$$
\begin{aligned}
\psi & =\frac{\pi}{2} \Longrightarrow \varphi=\frac{1}{2} \operatorname{atan}_{2}\left(r_{12}, r_{11}\right), \\
\theta & =\frac{\pi}{2} \\
\phi & =\pi
\end{aligned}
$$

and $\vartheta$ is no longer relevant as it has been already discussed.

\section{Quaternion Description of Precessions}

To derive the kinematic equations in quaternion terms, it suffices to recall that $\mathrm{c}=\zeta / \zeta_{0}$ and substitute it in formula (10), which yields upon multiplication by $\zeta_{0}^{2}$

$$
2 \zeta_{0} \dot{\zeta}-2 \dot{\zeta} \zeta=\zeta_{0}^{2} \Omega+(\zeta, \Omega) \zeta+\zeta_{0} \Omega^{\times} \zeta
$$

Then, taking a dot product with $\zeta$ and using the identities

$$
\|\zeta\|^{2}=\zeta_{0}^{2}+\zeta^{2}=1 \Longrightarrow \zeta_{0} \dot{\zeta}_{0}+(\zeta, \dot{\zeta})=0
$$

yield the derivative of the scalar (real) part of the quaternion

$$
2 \dot{\zeta}_{0}=-(\boldsymbol{\Omega}, \zeta)
$$

Combining both expressions, we obtain the vector (imaginary) part in the form

$$
2 \dot{\zeta}=\mathbf{\Omega} \times \zeta+\zeta_{0} \Omega,
$$

so the kinematic equations have the block-matrix representation

$$
\left(\begin{array}{c}
\dot{\zeta} \\
\dot{\zeta}_{0}
\end{array}\right)=\frac{1}{2}\left(\begin{array}{cc}
\boldsymbol{\Omega}^{\times} & \boldsymbol{\Omega} \\
-\boldsymbol{\Omega}^{t} & 0
\end{array}\right)\left(\begin{array}{l}
\zeta \\
\zeta_{0}
\end{array}\right) .
$$

Identifying quaternions and four-vectors allows for writing the above result as $\dot{\zeta}=\mathscr{A} \zeta$ and, thus, derive the general solution for the propagator in terms of time-ordered matrix exponents. In the particular case $\boldsymbol{\Omega}=$ const., however, we end up with a system of linear homogeneous ODEs with constant coefficients, hence the solution

$$
\zeta(t)=\mathrm{e}^{t \mathscr{A}} \zeta(0) .
$$

Note that $\operatorname{det}(\mathscr{A}-\lambda \mathscr{I})=\left(\lambda^{2}+\omega^{2}\right)^{2}$, so we have two double roots $\lambda_{ \pm}= \pm \mathrm{i} \omega$, which yields, in the canonical basis, synchronized rotations (with equal frequencies) in two mutually perpendicular planes in $\mathbb{R}^{4}$ as we have seen in the case $\Omega_{1,2}=0$. Moreover,

$$
\mathscr{A}^{2 k}=(-1)^{k} \omega^{2 k} \mathscr{I}, \quad \mathscr{A}^{2 k+1}=(-1)^{k} \omega^{2 k+1} \widehat{\mathscr{A}}
$$

and, thus, the propagator may be expressed as

$$
\mathrm{e}^{t \mathscr{A}}=\cos \omega t \mathscr{I}+\sin \omega t \widehat{\mathscr{A}}
$$

where we let $\mathscr{I}$ denote the identity in any dimension and $\widehat{\mathscr{A}}=$ $\omega^{-1} \mathscr{A}$ with $\omega=(1 / 2)|\Omega|$. Bearing this in mind, for the time evolution of an arbitrary quaternion $\zeta(t)$, one has

$$
\begin{aligned}
& \zeta(t)=\cos \omega t \dot{\zeta}+\sin \omega t\left(\dot{\zeta}_{0} \widehat{\boldsymbol{\omega}}+\widehat{\boldsymbol{\omega}} \times \dot{\zeta}\right), \\
& \zeta_{0}(t)=\dot{\zeta}_{0} \cos \omega t-(\dot{\boldsymbol{\zeta}}, \widehat{\boldsymbol{\omega}}) \sin \omega t,
\end{aligned}
$$

where $\widehat{\boldsymbol{\omega}}=(1 / 2 \omega) \boldsymbol{\Omega}$ stands for the unit vector in the direction of $\Omega$ and $\dot{\zeta}=\zeta(0)$ is simply the initial condition.

Denoting also $\stackrel{\circ}{\mathbf{c}}=\mathbf{c}(0)=\dot{\zeta} / \dot{\zeta}_{0}$, we obtain the time dependence of the vector-parameter

$$
\mathbf{c}(t)=\frac{\stackrel{\circ}{\mathbf{c}}+\tan \omega t\left(\mathscr{I}-\stackrel{\circ}{\mathbf{c}}^{\times}\right) \widehat{\boldsymbol{w}}}{1-(\stackrel{\circ}{\mathbf{c}}, \widehat{\boldsymbol{w}}) \tan \omega t} .
$$

In particular, if $\zeta(0)$ is parallel to $\widehat{\boldsymbol{\omega}}$, it remains such along the flow determined by (46) as one has

$$
\begin{aligned}
& \left(\begin{array}{l}
\zeta(t) \\
\zeta_{0}(t)
\end{array}\right) \\
& \quad=\left(\begin{array}{cc}
\cos \omega t \mathscr{I}+\sin \omega t \widehat{\boldsymbol{\omega}}^{\times} & \sin \omega t \widehat{\boldsymbol{\omega}} \\
-\sin \omega t \widehat{\boldsymbol{\omega}}^{t} & \cos \omega t
\end{array}\right)\left(\begin{array}{r}
\sin \dot{\vartheta} \widehat{\boldsymbol{\omega}} \\
\operatorname{oos} \dot{\vartheta}
\end{array}\right) \\
& =\left(\begin{array}{c}
\sin (\omega t+\dot{\vartheta}) \widehat{\boldsymbol{\omega}} \\
\cos (\omega t+\dot{\vartheta})
\end{array}\right),
\end{aligned}
$$

so we end up with the trivial orbit $\mathbf{c}(t)=\tan \vartheta \widehat{\boldsymbol{\omega}}$, which gives the solution also in the case of a steady initial state $\left(\dot{\zeta}_{0}=1\right)$. Similarly, if $\dot{\boldsymbol{\zeta}} \perp \widehat{\boldsymbol{\omega}}$ and $\dot{\zeta}_{0}=0$, one has $\mathbf{c}(t)=\stackrel{\circ}{\mathbf{c}}+\tan \omega t \widehat{\boldsymbol{\omega}} \times \stackrel{\circ}{\mathbf{c}}$, so $\mathbf{c}(t)$ remains perpendicular to $\widehat{\boldsymbol{\omega}}$. More generally, we may denote $\mathrm{p}(t)=(\mathbf{c}(t), \widehat{\boldsymbol{\omega}})$ and $\mathrm{p}(0)=\tan \stackrel{\circ}{\hat{\vartheta}}$, as well as $\mathbf{q}=\mathbf{c}-$ $\mathrm{p} \widehat{\boldsymbol{\omega}}=\mathscr{P}_{\widehat{\boldsymbol{\omega}}}^{\perp} \mathbf{c}$, and obtain

$$
\begin{aligned}
& \mathrm{p}(t)=\tan \vartheta(t), \\
& \mathbf{q}(t)=\frac{\mathscr{I}+\tan \omega t \widehat{\boldsymbol{\omega}}^{\times}}{1-\tan \stackrel{\circ}{\vartheta} \tan \omega t} \mathbf{q}(0) .
\end{aligned}
$$

Note that, just as before, we may weaken the condition of constant angular velocity by demanding only $\widehat{\boldsymbol{\omega}}=$ const., thus letting $\omega=\omega(t)$. This introduces just a slight modification of the above solution: namely, in formula (46), we make the substitution

$$
\omega t \longrightarrow \int_{0}^{t} \omega(s) \mathrm{d} s
$$

It is useful to point out that the matrix $\mathscr{A}$ (and therefore, its exponent) may be expressed also in a $2 \times 2$ block form. In particular, if $\widehat{\boldsymbol{\omega}}=(0,0,1)^{t}$, one has

$$
\mathrm{e}^{t \mathscr{A}}=\left(\begin{array}{cc}
\mathrm{e}^{\mathscr{f} \omega t} & 0 \\
0 & \mathrm{e}^{-\mathscr{f} \omega t}
\end{array}\right), \quad \mathscr{J}=\left(\begin{array}{cc}
0 & -1 \\
1 & 0
\end{array}\right)
$$

which yields the solutions obtained in the previous section. More generally, we have

$$
\widehat{\mathscr{A}}=\left(\begin{array}{cc}
\widehat{\omega}_{3} \mathscr{J} & \widehat{\omega}_{2} \mathscr{I}-\widehat{\omega}_{1} \mathscr{J} \\
-\widehat{\omega}_{2} \mathscr{I}-\widehat{\omega}_{1} \mathscr{J} & -\widehat{\omega}_{3} \mathscr{J}
\end{array}\right),
$$

that is, a real $4 \times 4$ representation of a pure unit quaternion and may be mapped onto $\mathfrak{g} \mathfrak{i t}(2)$ as $\widehat{\mathscr{A}} \rightarrow \widehat{\mathscr{A}}^{\star}=\widehat{\omega}_{3} \mathbf{i}+\widehat{\omega}_{2} \mathbf{j}-\widehat{\omega}_{1} \mathbf{k}$. The action of $\mathscr{A}^{\star}=\omega \widehat{\mathscr{A}}^{\star}$ on $\zeta$ is naturally realized via matrix multiplication if the latter is represented as a point $(\xi, \eta)$ in $\mathbb{C}^{2}$ : namely, 


$$
\begin{aligned}
& \xi=\zeta_{1}+\mathrm{i} \zeta_{2}, \\
& \eta=\zeta_{3}+\mathrm{i} \zeta_{0} .
\end{aligned}
$$

Then, the kinematic equation for $\zeta$ may be written as $\dot{\zeta}=\mathscr{A}^{\star} \zeta$, or in components

$$
\begin{aligned}
\left(\begin{array}{l}
\dot{\xi} \\
\dot{\eta}
\end{array}\right) & =\left(\begin{array}{cc}
\mathrm{i} \omega_{3} & \omega_{2}-\mathrm{i} \omega_{1} \\
-\omega_{2}-\mathrm{i} \omega_{1} & -\mathrm{i} \omega_{3}
\end{array}\right)\left(\begin{array}{l}
\xi \\
\eta
\end{array}\right), \\
\omega_{k} & =\omega \widehat{\omega}_{k}
\end{aligned}
$$

and for fixed $\widehat{\boldsymbol{\omega}}$ the corresponding unitary evolution operator takes the form

$$
\begin{aligned}
\exp \left(\int_{0}^{t} \omega(s) \mathrm{d} s \widehat{\mathscr{A}}^{\star}\right)= & \cos \int_{0}^{t} \omega(s) \mathrm{d} s \mathscr{I} \\
& +\sin \int_{0}^{t} \omega(s) \mathrm{d} s \widehat{\mathscr{A}}^{\star} .
\end{aligned}
$$

\section{Precessing Angular Velocity}

Let us now consider the case of varying directional vector $\widehat{\boldsymbol{\omega}}$. One relatively simple setting is that of an angular velocity precessing about a fixed axis: for example,

$$
\boldsymbol{\Omega}=2\left(\alpha \cos 2 \omega_{0} t, \alpha \sin 2 \omega_{0} t, \omega\right)^{t}, \quad \alpha, \omega, \omega_{0} \in \mathbb{R}^{+} .
$$

This time the magnitude $\Omega=2 \sqrt{\alpha^{2}+\omega^{2}}$ is constant, so one has

$$
\mathscr{A}^{\star}(t)=-\mathrm{i}\left(\begin{array}{cc}
-\omega & \alpha \mathrm{e}^{2 \mathrm{i} \omega_{0} t} \\
\alpha \mathrm{e}^{-2 \mathrm{i} \omega_{0} t} & \omega
\end{array}\right)
$$

and due to the nonvanishing commutator $\left[\mathscr{A}^{\star}(t)\right.$, $\left.\int_{0}^{t} \mathscr{A}^{\star}(s) \mathrm{d} s\right] \neq 0$, obtaining the propagator via exponentiating the above matrix generally involves time-ordering or some other cumbersome procedure. Nevertheless, one may still

$$
\mathcal{U}(t)=\left(\begin{array}{cc}
\mathrm{e}^{\mathrm{i} \omega_{0} t}\left(\cos \sigma t+\mathrm{i} \sin \psi_{0} \sin \sigma t\right) & -\mathrm{i} \mathrm{e}^{\mathrm{i} \omega_{0} t} \cos \psi_{0} \sin \sigma t \\
-\mathrm{i} \mathrm{e}^{-\mathrm{i} \omega_{0} t} \cos \psi_{0} \sin \sigma t & \mathrm{e}^{-\mathrm{i} \omega_{0} t}\left(\cos \sigma t-\mathrm{i} \sin \psi_{0} \sin \sigma t\right)
\end{array}\right)
$$

where for the first row we substitute the expressions for $A, B \sim$ $\dot{\xi}, \stackrel{\circ}{\eta}$ in the equation for $\xi(t)$, while for the second one, we use the fact that $\mathcal{U}(t)$ takes values in $\mathrm{SU}(2)$. Considering, for example, a steady initial state $(\dot{\xi}, \stackrel{\circ}{\eta})=(0, i)$ from the above, one easily obtains

$$
\begin{aligned}
& \zeta_{0}(t)=\cos \sigma t \cos \omega_{0} t-\sin \psi_{0} \sin \sigma t \sin \omega_{0} t, \\
& \zeta_{1}(t)=\cos \psi_{0} \sin \sigma t \cos \omega_{0} t, \\
& \zeta_{2}(t)=\cos \psi_{0} \sin \sigma t \sin \omega_{0} t, \\
& \zeta_{3}(t)=\sin \psi_{0} \sin \sigma t \cos \omega_{0} t+\cos \sigma t \sin \omega_{0} t .
\end{aligned}
$$

derive the exact solution transforming system (53) into a single ODE. Namely, after differentiating the first equation and substituting

$$
\alpha \eta=\mathrm{e}^{-2 \mathrm{i} \omega_{0} t}(\mathrm{i} \dot{\xi}+\omega \xi)
$$

from the second one, the time dependence of the coefficients factors out and we end up with a linear homogeneous secondorder ODE with constant coefficients

$$
\ddot{\xi}-2 \mathrm{i} \omega_{0} \dot{\xi}+\left(\alpha^{2}+\omega^{2}-2 \omega \omega_{0}\right) \xi=0 .
$$

With the notation

$$
\begin{aligned}
\sigma & =\sqrt{\alpha^{2}+\chi^{2}}, \\
\chi & =\omega-\omega_{0}, \\
\psi_{0} & =\arctan \frac{\chi}{\alpha},
\end{aligned}
$$

Here, $A$ and $B$ are complex constants determined by the initial conditions $\stackrel{\circ}{\xi}=\xi(0)$ and $\dot{\eta}=\eta(0)$ as

$$
\begin{aligned}
A & =\stackrel{\circ}{\xi}, \\
\mathrm{i} B & =\cos \psi_{0} \stackrel{\circ}{\eta}-\sin \psi_{0} \omega \stackrel{\xi}{\xi} .
\end{aligned}
$$

This allows for writing the propagator $\mathcal{U}(t):(\stackrel{\circ}{\xi}, \stackrel{\circ}{\eta})^{t} \rightarrow(\xi, \eta)^{t}$ in the simple form

This allows for expressing also the evolution of the vectorparameter in the form (see Figure 1)

$$
\begin{aligned}
\mathbf{c}(t)= & \frac{1}{1-\sin \psi_{0} \tan \sigma t \tan \omega_{0} t} \\
& \times\left(\begin{array}{c}
\cos \psi_{0} \tan \sigma t \\
\cos \psi_{0} \tan \sigma t \tan \omega_{0} t \\
\sin \psi_{0} \tan \sigma t+\tan \omega_{0} t
\end{array}\right) .
\end{aligned}
$$

Taking into account the fact that $1+\mathbf{c}^{2}=\sec ^{2} \sigma t \sec ^{2} \omega t$, one may express the entries of the corresponding rotation 

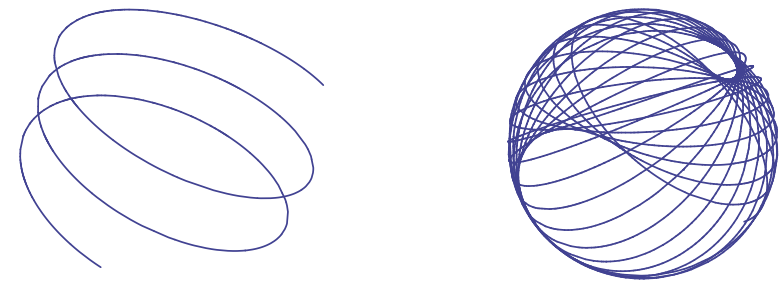

FIGURE 1: The evolution of the vector-parameter (65) and the trace of the corresponding rotation acting on the vector $(3,14,25)$.

matrix using formula (7). In particular, in the resonant limit $\omega_{0} \rightarrow \omega$, one has $\psi_{0}=0$ and $\sigma=\alpha$; hence, $\mathbf{c}(t)=(\tan \alpha t$, $\left.\tan \alpha t \tan \omega_{0} t, \tan \omega_{0} t\right)^{t}$, which finally yields

$$
\begin{aligned}
& \mathscr{R}(t)_{\chi=0} \\
& =\left(\begin{array}{ccc}
\cos 2 \omega_{0} t & -\cos 2 \alpha t \sin 2 \omega_{0} t & \sin 2 \alpha t \sin 2 \omega_{0} t \\
\sin 2 \omega_{0} t & \cos 2 \alpha t \cos 2 \omega_{0} t & -\sin 2 \alpha t \cos 2 \omega_{0} t \\
0 & \sin 2 \alpha t & \cos 2 \alpha t
\end{array}\right) .
\end{aligned}
$$

As we show in the next section, a similar expression holds also for variable $\alpha$ and $\omega_{0}$ (under certain restrictions) if we substitute $\alpha t \rightarrow \int_{0}^{t} \alpha(s) \mathrm{d} s$ and $\omega_{0} t \rightarrow \omega_{0}(t)$. One may also derive the rotation matrix using directly formula (4), written in components as

$$
\begin{aligned}
& \mathscr{R}(\zeta) \\
& =\left(\begin{array}{lll}
1-2\left(\zeta_{2}^{2}+\zeta_{3}^{2}\right) & 2\left(\zeta_{1} \zeta_{2}-\zeta_{0} \zeta_{3}\right) & 2\left(\zeta_{1} \zeta_{3}+\zeta_{0} \zeta_{2}\right) \\
2\left(\zeta_{1} \zeta_{2}+\zeta_{0} \zeta_{3}\right) & 1-2\left(\zeta_{1}^{2}+\zeta_{3}^{2}\right) & 2\left(\zeta_{2} \zeta_{3}-\zeta_{0} \zeta_{1}\right) \\
2\left(\zeta_{1} \zeta_{3}-\zeta_{0} \zeta_{2}\right) & 2\left(\zeta_{2} \zeta_{3}+\zeta_{0} \zeta_{1}\right) & 1-2\left(\zeta_{1}^{2}+\zeta_{2}^{2}\right)
\end{array}\right) .
\end{aligned}
$$

\section{Generic Angular Velocity}

Our construction may be generalized as follows: let us consider a time-varying angular velocity vector in the form (note once more that here $\Omega(t)=2 \sqrt{\omega^{2}+\alpha^{2}}$ )

$$
\boldsymbol{\Omega}(t)=2\left(\alpha(t) \cos 2 \omega_{0}(t), \alpha(t) \sin 2 \omega_{0}(t), \omega(t)\right)^{t} .
$$

We use a standard technique in physics to factor out the dependence on the diagonal part $\mathscr{A}_{0}^{\star}=\mathscr{A}_{\alpha=0}^{\star}$ of (56) known as the interaction picture (cf. [16]). It involves expressing the propagator as (the matrix $\mathscr{A}_{0}^{\star}$ is not necessarily constant; we only demand that it commutes with its integral; see [16])

$$
\mathscr{U}(t)=\mathscr{U}_{0}(t) \mathscr{U}_{I}(t), \quad \mathscr{U}_{0}(t)=\exp \int_{0}^{t} \mathscr{A}_{0}^{\star}(s) \mathrm{d} s,
$$

where $\mathcal{U}_{I}(t)$ solves the reduced equation (this is easy to see via straightforward differentiation)

$$
\dot{\zeta}_{I}(t)=\mathscr{A}_{I}^{\star}(t) \zeta_{I}(t), \quad \mathscr{A}_{I}^{\star}=\mathscr{U}_{0}^{-1} \mathscr{A}^{\star} \mathscr{U}_{0}-\mathscr{A}_{0}^{\star}, \quad \zeta_{I}=\mathscr{U}_{0}^{-1} \zeta .
$$

With the aid of formulas (53) and (68), we express

$$
\begin{aligned}
& \mathscr{U}_{0}(t)=\left(\begin{array}{cc}
\mathrm{e}^{\mathrm{i} \int_{0}^{t} \omega(s) \mathrm{d} s} & 0 \\
0 & \mathrm{e}^{-\mathrm{i} \int_{0}^{t} \omega(s) \mathrm{d} s}
\end{array}\right), \\
& \mathscr{A}_{I}^{\star}(t)=-\mathrm{i} \alpha\left(\begin{array}{cc}
0 & \mathrm{e}^{-2 \mathrm{i} \chi} \\
\mathrm{e}^{2 \mathrm{i} \chi} & 0
\end{array}\right)
\end{aligned}
$$

with the notation $\chi(t)=\int_{0}^{t} \omega(s) \mathrm{d} s-\omega_{0}(t)$. Note that in the special case $\chi=$ const. the matrix $\mathscr{A}_{I}^{\star}(t)$ commutes with its integral and may thus be exponentiated trivially. Then, the evolution operator $\mathcal{U}(t)=\mathscr{U}_{0}(t) \mathcal{U}_{I}(t)$ has the simple form

$$
\begin{aligned}
& \mathcal{U}(t) \\
& =\left(\begin{array}{cc}
\mathrm{e}^{\mathrm{i}\left(\omega_{0}+\chi\right)} \cos \int_{0}^{t} \alpha(s) \mathrm{d} s & -\mathrm{i} \mathrm{e}^{\mathrm{i}\left(\omega_{0}-\chi\right)} \sin \int_{0}^{t} \alpha(s) \mathrm{d} s \\
-\mathrm{i} \mathrm{e}^{-\mathrm{i}\left(\omega_{0}-\chi\right)} \sin \int_{0}^{t} \alpha(s) \mathrm{d} s & \mathrm{e}^{-\mathrm{i}\left(\omega_{0}+\chi\right)} \cos \int_{0}^{t} \alpha(s) \mathrm{d} s
\end{array}\right)
\end{aligned}
$$

and in particular, for $\chi=0$, the corresponding rotation matrix is reduced to

$$
\mathscr{R}(t)_{\chi=0}=\left(\begin{array}{ccc}
\cos 2 \omega_{0}(t) & -\sin 2 \omega_{0}(t) \cos 2 \int_{0}^{t} \alpha(s) \mathrm{d} s & \sin 2 \omega_{0}(t) \sin 2 \int_{0}^{t} \alpha(s) \mathrm{d} s \\
\sin 2 \omega_{0}(t) & \cos 2 \omega_{0}(t) \cos 2 \int_{0}^{t} \alpha(s) \mathrm{d} s & -\cos 2 \omega_{0}(t) \sin 2 \int_{0}^{t} \alpha(s) \mathrm{d} s \\
0 & \sin 2 \int_{0}^{t} \alpha(s) \mathrm{d} s & \cos 2 \int_{0}^{t} \alpha(s) \mathrm{d} s
\end{array}\right) .
$$

We conclude this section with a comment on the case of nonconstant $\chi(t)$, which yields some iterative procedure (such as time-ordered exponentiation) for obtaining the propagator in the interaction picture. Here we choose to work with a version of Magnus expansion, usually referred to as the continuous BCH formula (see [16]). Note that in the standard quaternion basis, we have the representation $\mathscr{A}_{I}^{\star}=u_{1} \mathbf{j}+u_{2} \mathbf{k}$ with

$$
\begin{aligned}
& u_{1}(t)=\alpha(t) \sin 2 \chi(t), \\
& u_{2}(t)=-\alpha(t) \cos 2 \chi(t),
\end{aligned}
$$

which allows us to calculate the corrections explicitly in some special cases. Consider, for example, small precessions about the $z$-axis with $\alpha=2 \varepsilon\left(\dot{\omega}_{0}-\omega\right)=-2 \varepsilon \dot{\chi}$, where $\varepsilon \ll 1$ is regarded as a perturbation parameter. A straightforward computation yields 


$$
\begin{aligned}
\mathscr{U}_{I}(t) \approx & \left(1-\varepsilon^{2} \sin ^{2} \Delta \chi\right) \mathscr{I}+\varepsilon^{2}(\sin 2 \Delta \chi-2 \Delta \chi) \mathbf{i} \\
& +\left.\varepsilon \cos 2 \chi\right|_{0} ^{t} \mathbf{j}+\left.\varepsilon \sin 2 \chi\right|_{0} ^{t} \mathbf{k},
\end{aligned}
$$

where we ignore terms of order $\varepsilon^{3}$ and use the notation $\Delta \chi(t)=\chi(t)-\chi(0)$. At the same time, $\mathscr{U}_{0}(t)$ remains as before and we still have the expression $\mathcal{U}(t)=\mathscr{U}_{0}(t) \mathcal{U}_{I}(t)$.

\section{A Note on Dynamics}

Consider the Euler equations describing free rigid body rotation (cf. [17])

$$
\begin{aligned}
& \dot{\Omega}_{1}=\mu_{1} \Omega_{2} \Omega_{3}, \\
& \dot{\Omega}_{2}=\mu_{2} \Omega_{1} \Omega_{3}, \\
& \dot{\Omega}_{3}=\mu_{3} \Omega_{1} \Omega_{2},
\end{aligned}
$$

where the constants $\mu_{k}$ are expressed in terms of the eigenvalues $I_{k}$ of the inertial tensor as

$$
\begin{aligned}
& \mu_{1}=\frac{I_{2}-I_{3}}{I_{1}}, \\
& \mu_{2}=\frac{I_{3}-I_{1}}{I_{2}}, \\
& \mu_{3}=\frac{I_{1}-I_{2}}{I_{3}} .
\end{aligned}
$$

It is interesting to view our previous considerations on the kinematics determined by a particular choice of angular velocity from a dynamical perspective. We shall further assume that the axes chosen in the parameterization of $\boldsymbol{\Omega}(t)$ in formula (68) coincide with the principal axes of the corresponding inertial ellipsoid. To begin with, it is almost straightforward to show that if the direction of the angular velocity given by the unit vector $\widehat{\boldsymbol{\omega}}$ is preserved, then its magnitude $\Omega$ is constant as well. For a free rotation described by formula (63), on the other hand, the Euler dynamical equations (76) yield a symmetric inertial ellipsoid with $I_{1}=$ $I_{2}=I$ and $I_{3}=\left(1+\omega_{0} / \omega\right) I$. In particular, in the limit $\sigma \rightarrow 0$, the solution (63) obviously converges to the one for the case $\Omega=$ const. and $\widehat{\boldsymbol{\omega}}=(0,0,1)^{t}$ as it should be expected. However, considering the general setting (68), we may express (76) in the form

$$
\begin{array}{r}
\dot{\alpha}=2 \alpha \tan 2 \omega_{0}\left(\mu_{1} \omega+\dot{\omega}_{0}\right)=2 \alpha \cot 2 \omega_{0}\left(\mu_{2} \omega-\dot{\omega}_{0}\right), \\
\dot{\omega}=\mu_{3} \alpha^{2} \sin 4 \omega_{0} .
\end{array}
$$

Further on, we easily derive

$$
\dot{\omega}_{0}(t)=\omega(t)\left(\mu_{2} \cos ^{2} 2 \omega_{0}(t)-\mu_{1} \sin ^{2} 2 \omega_{0}(t)\right),
$$

so in the particular case of rotationally symmetric inertial ellipsoid with $\mu_{2}=-\mu_{1}=\mu$ and $\mu_{3}=0$, we have $\dot{\omega}_{0}=\mu \omega$, but then the third equality yields $\omega=$ const., so $\omega_{0}=\mu \omega t$ up to an additive phase. Finally, substituting into the equation for $\alpha(t)$, we find that $\alpha=$ const. as well, so the solution is a linear precession with frequency $2 \omega_{0}$ about the $z$-direction, for which the angular velocity magnitude $\Omega=2 \sqrt{\alpha^{2}+\omega^{2}}$ is also constant. On the other hand, we may assume only $\alpha=$ const., which immediately leads to $\mu_{2}=-\mu_{1}$ and due to the triangle inequality for the principal moments of inertia, this inevitably yields $\mu_{3}=0$, but then $\dot{\omega}=0$ and we obtain the previously considered case once more.

Let us now investigate the setting $\mu_{1}=\mu_{2}=\mu$, which immediately yields the relations

$$
\begin{gathered}
I_{3}=\frac{I_{1}^{2}+I_{2}^{2}}{I_{1}+I_{2}}, \\
\mu=\frac{I_{2}-I_{1}}{I_{2}+I_{1}}, \\
\mu_{3}=\frac{I_{1}^{2}-I_{2}^{2}}{I_{1}^{2}+I_{2}^{2}} .
\end{gathered}
$$

This symmetry reduces system (78) to

$$
\begin{aligned}
\dot{\alpha} & =2 \mu \alpha \omega \sin 4 \omega_{0}, \\
\dot{\omega} & =\mu_{3} \alpha^{2} \sin 4 \omega_{0}, \\
\dot{\omega}_{0} & =\mu \omega \cos 4 \omega_{0}
\end{aligned}
$$

and we readily find a conservation law in the form

$$
2 \mu \omega^{2}-\mu_{3} \alpha^{2}=C
$$

Choosing further $\stackrel{\circ}{\alpha}=\alpha(0)$ allows us to obtain also

$$
\begin{aligned}
& \alpha(t)=\stackrel{\circ}{\alpha} \sqrt{\left|\sec 4 \omega_{0}\right|}, \\
& \omega(t)= \pm \frac{1}{\sqrt{2 \mu}}\left(C+\mu_{3} \stackrel{\circ}{\alpha}^{2}\left|\sec 4 \omega_{0}\right|\right)^{1 / 2}, \\
& \int \mathrm{d} t= \pm \sqrt{\frac{2}{\mu}} \int \frac{\sec 4 \omega_{0} \mathrm{~d} \omega_{0}}{\left(C+\mu_{3} \stackrel{\circ}{\alpha}^{2}\left|\sec 4 \omega_{0}\right|\right)^{1 / 2}} .
\end{aligned}
$$

However, the above solution does not provide explicit expressions for the propagator as it yields $\dot{\omega}_{0}(t) \neq \omega(t)$. A natural way to reach beyond the trivial setting of linear precession and still be able to use formula (72) is to introduce external forces (whose resultant moment is denoted with $\mathbf{f}$ ) to the Euler dynamical system (76). Let us consider the simple case of rotational inertial ellipsoid $I_{1}=I_{2}=I$, that is, $\mu_{2}=-\mu_{1}=$ $\mu \Rightarrow \mu_{3}=0$, as it allows for arranging $\chi=$ const. in a straightforward manner and, thus, reconstruct the rotation matrix from the angular velocity vector using expressions (72) and (4). Namely, we have $\dot{\omega}_{0}(t)=\omega(t)$, that is, $\chi=$ const., as long as we set $\mu=1$ and $\mathrm{f}_{2}=\mathrm{f}_{1} \tan 2 \omega_{0}$. Then, the solution takes the simple form 


$$
\begin{aligned}
\alpha(t) & =\alpha(0)+\frac{1}{2 I} \int_{0}^{t} \tilde{\mathrm{f}}(s) \mathrm{d} s, \\
\omega(t) & =\omega(0)+\frac{1}{2 I_{3}} \int_{0}^{t} \mathrm{f}_{3}(s) \mathrm{d} s, \\
\omega_{0}(t) & =\int_{0}^{t} \omega(s) \mathrm{d} s-\chi
\end{aligned}
$$

where $\tilde{\mathrm{f}}=\mathrm{f}_{1} \sec 2 \omega_{0}$ and in particular, setting $\mathrm{f}_{2}=\mathrm{f}_{1}=0$, we end up with a precessional motion with $\alpha=$ const., while $\omega(t)$ and $\omega_{0}(t)$ can still be determined from (84).

\section{Larmor Precession and Rabi Cycles}

The quaternion representation of the kinematical problem described above has many advantages, as we could see. One of them, which we have not discussed so far, is that it allows for a straightforward implementation of our solutions to other physical models governed by analogous equations. One particularly famous example appears in the context of Pauli's description of spin $1 / 2$ particles in an external magnetic field $B$, for which the kinematics of the spin state $\Psi$ is determined by the equation

$$
\dot{\Psi}=\frac{\gamma}{2} B \Psi
$$

where $\gamma$ is a physical constant (the gyromagnetic ratio) and the magnetic induction $B$ may be written as a purely imaginary quaternion, so the evolution of the above system describes a rotor with angular velocity $\boldsymbol{\Omega}=\gamma \mathbf{B}$. Then, we may use our solutions (with the proper rescaling) in a completely different physical context: for the description of spin precession in a coaxial (54) or a precessing magnetic field. The former is a famous quantum mechanical effect known as Larmor precession, while the latter was considered previously by Rabi in [18]. His solution (that won him a Nobel prize back in 1944) has a crucial impact on magnetic resonance imaging, quantum computing, and optics. A major part of the idea is based on the so-called Rabi resonance, which yields the highest probability for a transition from a $\downarrow$ to a $\uparrow$ spin state. The resonance corresponds to our solution with $\chi=0$, which may be interpreted also as a decomposability condition for $\mathcal{U}(t)$ into a pair of orthogonal pure quaternions

$$
\begin{aligned}
\mathcal{U}(t) & =\left(\begin{array}{cc}
\mathrm{e}^{\mathrm{i} \omega t} \cos \alpha t & -\mathrm{i} \mathrm{e}^{\mathrm{i} \omega t} \sin \alpha t \\
-\mathrm{ie}^{-\mathrm{i} \omega t} \sin \alpha t & \mathrm{e}^{-\mathrm{i} \omega t} \cos \alpha t
\end{array}\right) \\
& =\left(\begin{array}{cc}
\mathrm{e}^{\mathrm{i} \omega t} & 0 \\
0 & \mathrm{e}^{-\mathrm{i} \omega t}
\end{array}\right)\left(\begin{array}{cc}
\cos \alpha t & -\mathrm{i} \sin \alpha t \\
-\mathrm{i} \sin \alpha t & \cos \alpha t
\end{array}\right) .
\end{aligned}
$$

With the initial condition $(\stackrel{\circ}{\xi}, \stackrel{\circ}{\eta})=(0, i)$, this yields also the decomposition

$$
\mathscr{R}(\mathbf{c})=\mathscr{R}_{z}(2 \omega t) \mathscr{R}_{x}(2 \alpha t)
$$

into successive precessions about the $O X$ and the $O Z$ axis, with angular velocities $2 \alpha$ and $2 \omega$, respectively. Finally, one may consider solution (72) corresponding to a varying magnetic field satisfying only the condition $\chi=$ const., which may be shown (at least perturbatively) to provide the Rabi resonance in this more general case. On the other hand, a decomposition of the above type is possible only for $\chi=0$, which yields

$$
=\left(\begin{array}{cc}
\mathrm{e}^{\mathrm{i} \omega_{0}} & 0 \\
0 & \mathrm{e}^{-\mathrm{i} \omega_{0}}
\end{array}\right)\left(\begin{array}{cc}
\cos \int_{0}^{t} \alpha(s) \mathrm{d} s & -\mathrm{i} \sin \int_{0}^{t} \alpha(s) \mathrm{d} s \\
-\mathrm{i} \sin \int_{0}^{t} \alpha(s) \mathrm{d} s & \cos \int_{0}^{t} \alpha(s) \mathrm{d} s
\end{array}\right)
$$

and in particular, the transition probability is measured as $P_{\uparrow}=\left|\mathcal{U}_{12}\right|^{2}=\sin ^{2} \int_{0}^{t} \alpha(s) \mathrm{d} s$. For $\alpha=$ const. the time average is clearly given by $\left\langle P_{\uparrow}\right\rangle=1 / 2$. The choice of $\alpha(t)$ and $\omega(t)$ allows for a very precise control over the spin of the corresponding two-level system (qubits) and they will probably find good use in quantum computation (see, for example., [19]).

\section{Concluding Remarks}

Let us note that our representation $\Omega$ in the standard quaternion basis yields $\Omega \rightarrow \mathscr{A}^{\star}=\omega_{3} \mathbf{i}+\omega_{2} \mathbf{j}-\omega_{1} \mathbf{k} \in \mathfrak{g} \mathfrak{t}(2)$, since we chose to relate $\omega_{3}$ to the Cartan subalgebra. On the other hand, one may use the ordering $\zeta \rightarrow\left(\zeta_{0}, \zeta_{1}, \zeta_{2}, \zeta_{3}\right)^{t}$ or, equivalently, choose $(z, w)=\left(\zeta_{0}+\mathrm{i} \zeta_{1}, \zeta_{2}+\mathrm{i} \zeta_{3}\right)$, and then the quaternion associated with $\Omega$ may be written simply as $\mathscr{A}^{\star}=$ $\omega_{1} \mathbf{i}+\omega_{2} \mathbf{j}+\omega_{3} \mathbf{k}$. This choice of coordinates has its obvious advantages: for example, the propagator $\mathcal{U}(t)$ is a solution to the first-order scalar (in quaternion terms) initial value problem

$$
\dot{\zeta}=\mathscr{A}^{\star} \zeta, \quad \zeta(0)=\mathscr{I},
$$

where the quaternion $\zeta$ is expressed in the above basis as $\zeta=\zeta_{0} \mathbf{1}+\zeta_{1} \mathbf{i}+\zeta_{2} \mathbf{j}+\zeta_{3} \mathbf{k}$. In our representation, this clear picture is somehow distorted, but it agrees well with the classical description of the symmetric top or the usual treatment of the spin in quantum mechanics, which has its own merits. We also point out that both the perturbation technique and the dynamical considerations may be applied also to the vectorparameter description of the problem, but the quaternion realization here reduces the Riccati equations (10) to linear ones, which provides a serious simplification.

One major advantage of the above approach is simplicity (compared, for example, with [15]), which usually allows for a straightforward generalization of the results. In this particular case, one may easily extend the field of scalars from $\mathbb{R}$ to $\mathbb{C}$, preserving much of the obtained so far, but of course, dealing with new issues, such as isotropic directions. This exercise is not utterly pointless since the proper Lorentz group in special relativity is known to be isomorphic to the complex orthogonal group in dimension three: that is, $\operatorname{SO}(3, \mathbb{C}) \cong \operatorname{SO}^{+}(3,1)$. Thus, electromagnetic and relativistic effects such as Thomas precession, for instance, can be modeled in the complexified rigid body framework (see [13]). Another nontrivial step towards generalization would be considering the dual analogue 
of the above results in the context of nonhomogeneous Euclidean isometries and screw kinematics (cf. [20]). Finally, one may also focus on the applications in optics, nuclearmagnetic resonance, and quantum computation. However, a detailed treatment of each of these subjects alone would be a volume-demanding task; therefore, we leave such considerations for future research.

\section{Conflicts of Interest}

The authors declare that there are no conflicts of interest regarding the publication of this paper.

\section{References}

[1] C. Mladenova, "An Approach to Description of a Rigid Body Motion," Comptes rendus de l'Academie bulgare des Sciences, vol. 38, pp. 1657-1660, 1985.

[2] E. Piña, "Rotations with Rodrigues' vector," European Journal of Physics, vol. 32, no. 5, pp. 1171-1178, 2011.

[3] J. Kuipers, Quaternions and Rotation Sequences: A Primer with Applications to Orbits, Aerospace and Virtual Reality, Princeton University Press, Princeton, NJ, USA, 1999.

[4] S. Adler, Quaternionic Quantum Mechanics and Quantum Fields, Oxford University Press, New York, NY, USA, 1995.

[5] H. Hassanabadi, H. Sobhani, and A. Banerjee, "Relativistic scattering of fermions in quaternionic quantum mechanics," The European Physical Journal C, vol. 77, pp. 581-585, 2017.

[6] A. Sudbery, "Quaternionic analysis," Mathematical Proceedings of the Cambridge Philosophical Society, vol. 85, no. 2, pp. 199224, 1979.

[7] V. Kassandrov, "Biquaternion electrodynamics and weyl-cartan geometry of space-time," Gravitation and Cosmology, vol. 3, pp. 216-222, 1995.

[8] F. Fedorov, The Lorentz Group, Science, Moscow 1979.

[9] O. A. Bauchau and L. Trainelli, "The vectorial parameterization of rotation," Nonlinear Dynamics, vol. 32, no. 1, pp. 71-92, 2003.

[10] D. Brezov, C. Mladenova, and I. Mladenov, "Vector parameters in classical hyperbolic geometry," Journal of Geometry and Symmetry in Physics, vol. 30, pp. 19-48, 2013.

[11] I. Mladenov, "On the Saxon-Hutner theorem," Comptes rendus de l’Academie bulgare des Sciences, vol. 38, pp. 993-996, 1985.

[12] S. De Leo, "Quaternions and special relativity," Journal of Mathematical Physics, vol. 37, no. 6, pp. 2955-2968, 1996.

[13] D. S. Brezov, C. D. Mladenova, and I. M. Mladenov, "Wigner rotation and Thomas precession: geometric phases and related physical theories," Journal of the Korean Physical Society, vol. 66, no. 11, pp. 1656-1663, 2015.

[14] C. D. Mladenova, "Group-theoretical methods in manipulator kinematics and symbolic computations," Journal of Intelligent \& Robotic Systems, vol. 8, no. 1, pp. 21-34, 1993.

[15] O. M. O'Reilly and S. Payen, "The attitudes of constant angular velocity motions," International Journal of Non-Linear Mechanics, vol. 41, no. 6-7, pp. 787-795, 2006.

[16] S. Blanes, F. Casas, J. A. Oteo, and J. Ros, “The Magnus expansion and some of its applications," Physics Reports, vol. 470, no. 5-6, pp. 151-238, 2009.

[17] V.Arnold, Mathematical Methods of Classical Mechanics, Springer, New York, NY, USA, 1978.
[18] I. I. Rabi, "Space quantization in a gyrating magnetic field," Physical Review A: Atomic, Molecular and Optical Physics, vol. 51, no. 8, pp. 652-654, 1937.

[19] E. Kyoseva and N. V. Vitanov, "Arbitrarily accurate passband composite pulses for dynamical suppression of amplitude noise," Physical Review A: Atomic, Molecular and Optical Physics, vol. 88, no. 6, Article ID 063410, 2013.

[20] J. Wittenburg, Kinematics: Theory and Applications, Springer, Berlin, Germany, 2016. 


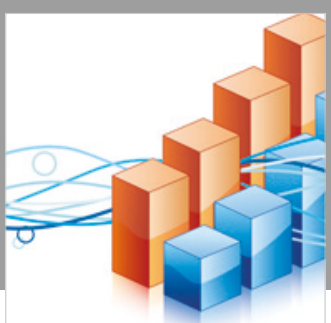

Advances in

Operations Research

\section{-n-m}
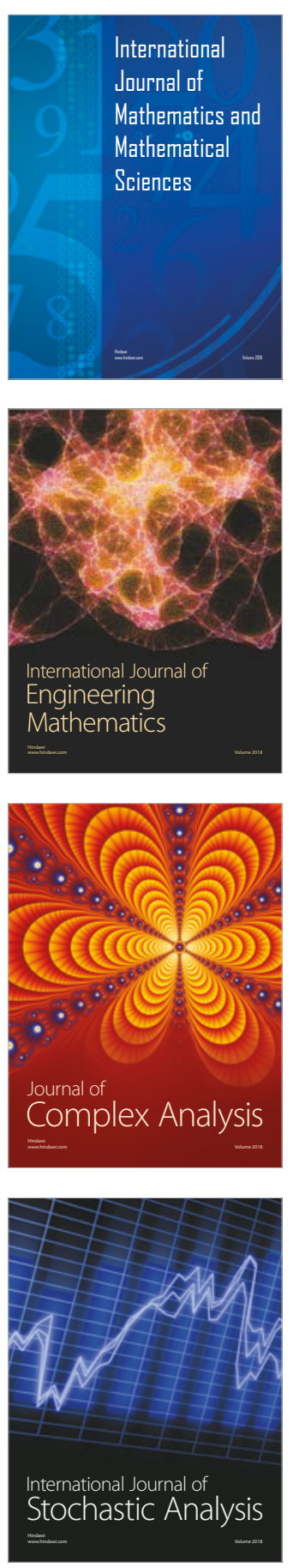
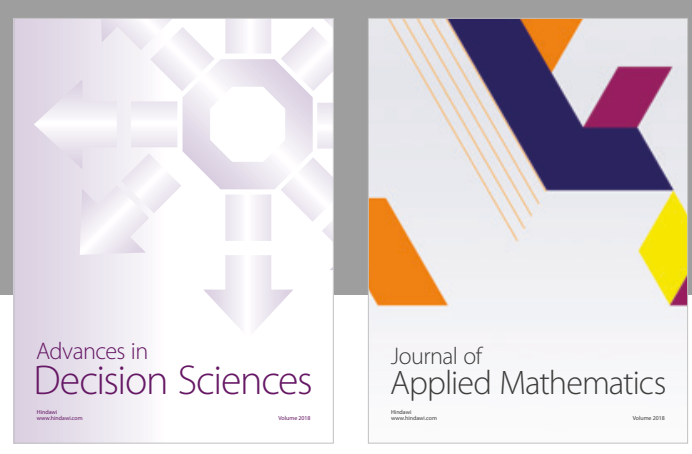

Journal of

Applied Mathematics
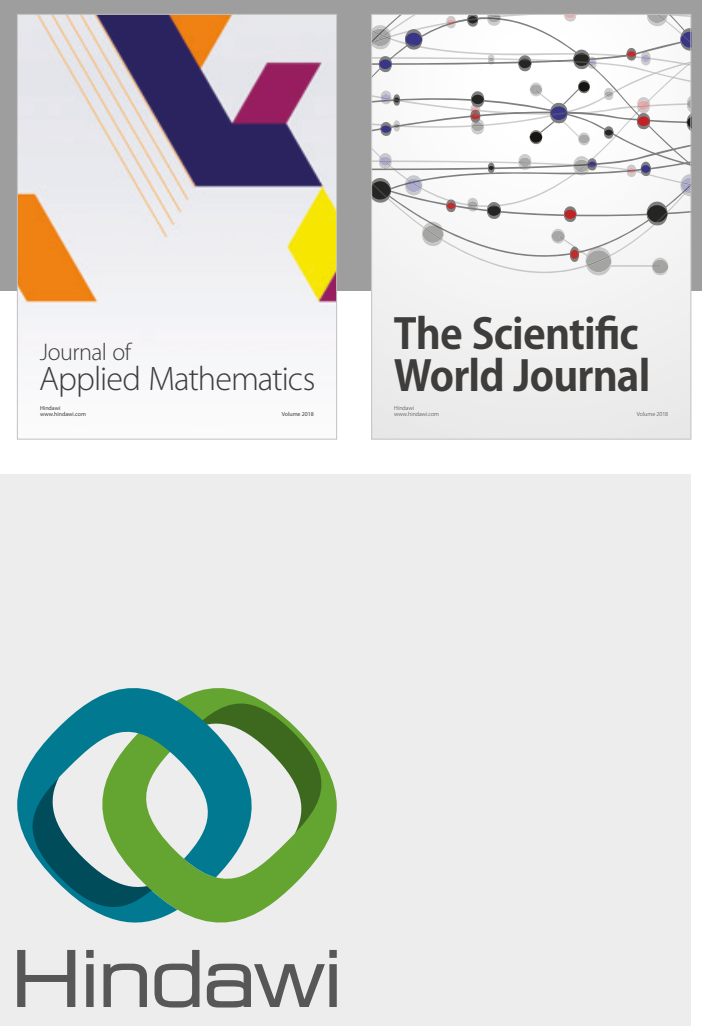

Submit your manuscripts at

www.hindawi.com

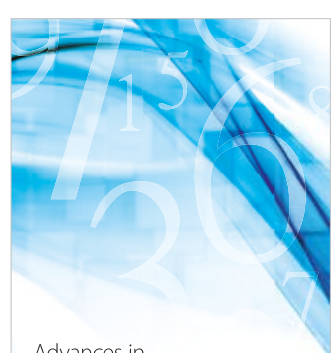

Advances in
Numerical Analysis
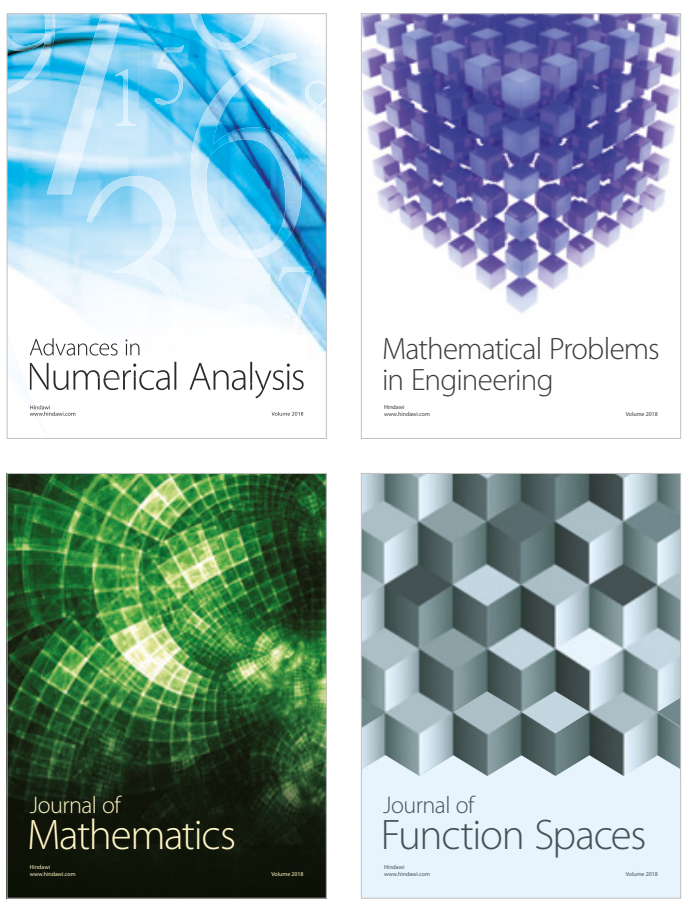

Mathematical Problems in Engineering

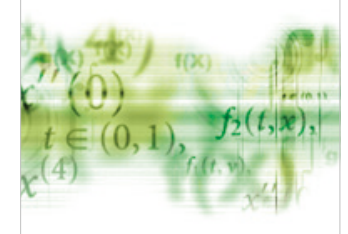

International Journal of

Differential Equations

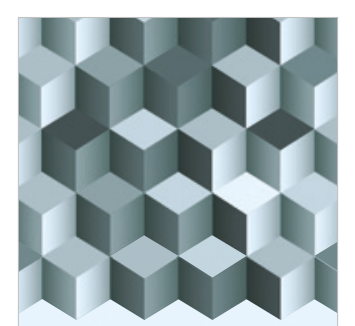

Journal of

Function Spaces

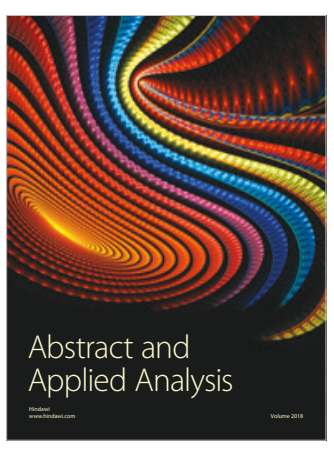

The Scientific

World Journal

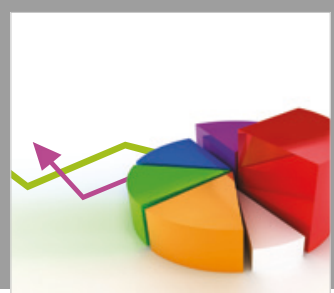

Journal of

Probability and Statistics
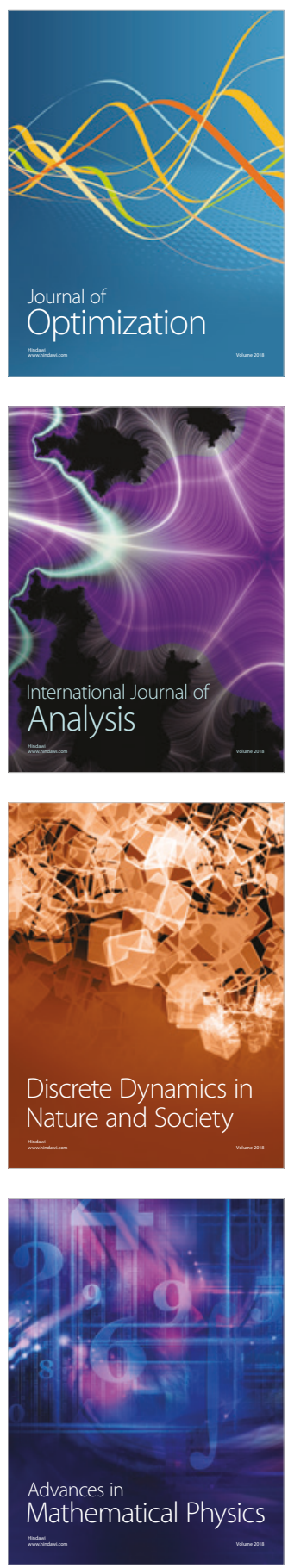